\title{
Liver Findings in Patients with Hereditary Hemorrhagic Telangiectasia
}

\author{
Wenyan Song, ${ }^{1,}$ Dawei Zhao, ${ }^{1,}{ }^{*}$ Hongjun Li, ${ }^{1}$ Jinli Ding, ${ }^{1}$ Ning He, ${ }^{1}$ and Yu Chen ${ }^{2}$ \\ ${ }^{1}$ Department of Radiology, Beijing YouAn Hospital, Capital Medical University, Beijing, China \\ ${ }^{2}$ Beijing Artificial Liver Treatment and Training Center, Beijing YouAn Hospital, Capital Medical University, Beijing, China \\ "Corresponding authors: Wenyan Song, Department of Radiology, Beijing YouAn Hospital, Capital Medical University, Beijing, China. Tel: +86-13611096669, E-mail: \\ 13611096669@163.com; Dawei Zhao, Department of Radiology, Beijing YouAn Hospital, Capital Medical University, Beijing, China, E-mail: zhaodw8999@163.com
}

Received 2015 June 29; Revised 2015 August 13; Accepted 2015 September 07.

\begin{abstract}
Background: Hereditary hemorrhagic telangiectasia (HHT), also known as Osler-Weber-Rendu syndrome, is a rare autosomal dominant genetic vascular dysplasia.

Objectives: The aim is to characterize the liver involvement of HHT by multi-detector helical computed tomography.

Patients and Methods: Through the use of multi-detector helical computed tomography, scan data derived from 12 cases of HHT were retrospectively studied, and an abdominal scan was performed on all of the patients.

Results: (i) Three types of shunting were found in the livers, including arteriovenous (hepatic artery to hepatic vein) in 6 cases, arterioportal (hepatic artery to portal vein) in 2 cases, and portal venous (portal vein to hepatic vein) in 4 cases, (ii) Biliary diseases were detected in 8 cases, including 3 cases of bilomas accompanied by increases of alkaline phosphatase (mean 271 units, ranging from 152 to 479 units) and arteriovenous shunts, and the other 5 cases revealed slight biliary dilation with no increase of alkaline phosphates, but were instead accompanied by arteriovenous shunts (3 cases) and portal venous shunts (2 cases). (iii) Vascular anatomic variants were observed in 4 cases, including 3 with accompanying arteriovenous shunts and 1 with accompanying arterioportal shunting. Conclusion: The involved liver with HHT typically shows vascular shunting and biliary diseases. Also, arteriovenous shunts may be vulnerable to biliary diseases.
\end{abstract}

Keywords: hereditary Hemorrhagic Telangiectasia, Liver Involvement, Vascular Dysplasia, Biliary Diseases

\section{Background}

Hereditary hemorrhagic telangiectasia (HHT), also known as Osler-Weber-Rendu syndrome, is a rare autosomal dominant genetic vascular dysplasia with an estimated prevalence of 1 to 2 cases per $10,000(1,2)$. Multiple organs can be involved in HHT; however, the clinical symptoms may have no specificity, which generally delays the diagnosis and treatment of HHT. Liver involvement is more common with a previous incidence of $8-31 \%$, which has been increased to about 74 - 79\% (3-5). Liver involvement in patients with HHT has not been fully characterized. The rapidly developing imaging technology, in particularly the multidetector computed tomography (MDCT), effectively facilitates the diagnosis of HHT. Although there is some previous literature discussing liver involvement of HHT, it is still rare to see a comprehensive discussion that also addresses both intrahepatic shunts and biliary diseases, especially the dynamic alteration of biloma via high quality CT images.

\section{Objectives}

The purpose of the present study is to characterize the liver involvement of HHT via CT images in conjunction with intrahepatic shunts.

\section{Patients and Methods}

\subsection{Clinical Data}

Between January of 2009 and June of 2014, a group of 12 patients with liver involvement of HHT in our hospital underwent dynamic contrast enhanced CT, and the scans were reviewed retrospectively. These 12 patients (including 11 women and 1 man), were aged between $42-68$ years, with a mean of 54.8 years. The clinical manifestations included repeated epistaxis, right upper quadrant abdominal pain, liver dysfunction, abdominal distension, fatigue, and loss of appetite. 


\subsection{Confirmed Diagnosis of HHT}

The patients with chronic liver disease and tumor-like lesions, such as hepatitis B, hepatitis $\mathrm{C}$, autoimmune liver disease, alcoholic liver disease, drug-induced liver disease, hemangioma, and primary liver cancer were all excluded from our study. The diagnosis of HHT requires the presence of at least three of the following criteria (6): (i) recurrent spontaneous epistaxis; (ii) mucocutaneous telangiectasia; (iii) a family medical history; and (iv) the presence of visceral involvement. All 12 patients were diagnosed with HHT since they all met at least three of the criteria. The clinical data and medical history of the 12 patients are shown in Table 1. In this study, the visceral involvement included the liver, lung, pancreas, spleen, and small intestine were detected by MDCT.

\subsection{Scanning}

Dynamic contrast enhanced CT was performed on all of the patients. Sixty four-section spiral CT (GE Light-Speed VCT, Milwaukee, WI, USA) was performed on the region of clinical interest with patients in the supine position with the following parameters: section thickness of $5 \mathrm{~mm}$, intervals of $5 \mathrm{~mm}$, rotation time of 0.5 seconds, $120 \mathrm{kV}$, and 380 $\mathrm{mAs}$. Section thickness and spacing of reconstruction were $0.625 \mathrm{~mm}$. An intravenous injection of nonionic contrast medium (iodine amine, $370 \mathrm{mg} \mathrm{I} / \mathrm{mL}, 100 \mathrm{ml}$ ) via the cubital vein was provided for the contrast enhanced scanning at a flow rate of $3 \mathrm{ml} / \mathrm{s}$. The scanning of the arterial phase, the venous phase, and the delayed phase were approximately 20s 25 s, 60s 70 s, and 180 s, respectively. Maximal intensity projection (MIP) images directly from the CT console for the three phases were reconstructed using a 0.625 $\mathrm{mm}$ interval, and the image data were sent to the workstation (GE ADW 4.3) for review and analysis. Two radiologists with 10 and 18 years of experience in abdominal and vascular imaging, respectively, reviewed the original images and reconstructions at the workstation. They were blind to the clinical data, and a consensual interpretation was reached by discussion. The radiologists also evaluated the presence and type of shunts.

\section{Results}

\subsection{Visceral Involvements}

Among all of the patients, 4 had cases of single liver involvement, and 8 had cases accompanied by other organ involvement including the pancreas ( 5 cases), lung involvement ( 3 cases), the spleen ( 1 case), and the small intestine (1 case).

\subsection{Scanning Findings}

Liver enlargement was observed by MDCT scanning, and intrahepatic anomalies during the contrast enhanced scanning could be detected (Table 2 ).

\subsubsection{Hepatic Vascular Anomaly \\ 4.2.1.1. Intrahepatic Shunts}

(i) An arteriovenous shunt was present in 6 cases (50\%). Dilation and tortuosity of the hepatic arteries and early opacification of the main hepatic veins could be observed in the arterial phase (Figure $1 \mathrm{~A}$ and $1 \mathrm{~B}$ ). The diameters of the hepatic arteries ranged from $8 \mathrm{~mm} \sim 11 \mathrm{~mm}$. Some large confluent vascular masses and several small telangiectasia were also detected (Figure $1 \mathrm{~A}$ and $1 \mathrm{C}$ ). (ii) An arterioportal shunt was found in 2 cases. Early opacification of the portal vein was detected during the arterial phases in the contrast-enhanced CT scans (Figure 2A), and tubular or nodular capillaries were observed around the hepatic periphery(Figure 2B).(iii)A portal venous shunt was detected in 4 cases. A dilated portal vein and a hepatic vein were revealed during the venous phase, and a focal vascular mass was found between the portal vein and hepatic vein (Figure 3A). Oblique MIP images more precisely showed the lesions (Figure 3B).

\subsubsection{Telangiectasia}

Telangiectasia was detected during the arterial phases in 8 patients. The vascular ectasias appeared to be round, with a diameter of $5-7 \mathrm{~mm}$ and a prevalently peripheral arrangement (Figure 1C and 2B). Diffuse and focal distributions were present in 6 cases and 2 cases, respectively.

\subsubsection{Large Confluent Vascular Masses}

Large confluent vascular masses were detected during the arterial phases, with diameters ranging from 5 to 20 $\mathrm{mm}$ (Figure $1 \mathrm{C}$ and $3 \mathrm{~A}$ ).

\subsubsection{Biliary Disease}

Biliary diseases were found in 8 patients, with 3 cases showing multiple round or tubular low density lesions with ill-defined boundaries and no enhancement in liver parenchyma in the MDCT images (Figure 4A). One of the 3 patients, who was suspected of having a liver abscess, received drainage treatment. No significant changes were shown after routine treatment, and the drained fluid was confirmed to be bile without bacteria. However, in another patient, an intrahepatic cyst grew in size after 8 months without any treatment (Figure 4B). These 3 patients showed elevated alkaline phosphatase levels (ALP, mean value of 271 per unit, ranging from 151 to 499 per 
Table 1. Clinical Data and Medical History of the 12 Patients

\begin{tabular}{|c|c|c|c|c|c|c|}
\hline No. & Gender & Age & Epistaxes & Telangiectasia & Family History & Visceral Involvement \\
\hline $\mathbf{1}$ & $\mathrm{F}$ & 55 & + & + & + & Liver \\
\hline 2 & $\mathrm{~F}$ & 60 & + & + & - & Liver \\
\hline 3 & $\mathrm{~F}$ & 60 & + & - & + & Liver, Pancreas \\
\hline 4 & $\mathrm{~F}$ & 45 & + & - & + & Liver \\
\hline 5 & $\mathrm{~F}$ & 46 & + & + & + & Liver, Pancreas, Small Intestine \\
\hline 6 & $\mathrm{~F}$ & 54 & + & + & + & Liver, Lung, Pancreas \\
\hline 7 & $\mathrm{~F}$ & 59 & + & - & + & Liver, Pancreas \\
\hline 8 & $\mathrm{~F}$ & 56 & + & + & - & Liver, Lung \\
\hline 9 & M & 42 & + & + & - & Liver, Spleen \\
\hline 10 & $\mathrm{~F}$ & 52 & + & + & + & Liver, Lung \\
\hline 11 & $\mathrm{~F}$ & 68 & + & + & + & Liver \\
\hline 12 & $\mathrm{~F}$ & 54 & + & + & + & Liver, Pancreas \\
\hline
\end{tabular}

Abbreviation: $\mathrm{F}$, female, $\mathrm{M}$, male.

Table 2. Liver Involvement in the 12 Patients by Scans

\begin{tabular}{|c|c|c|c|c|c|}
\hline No. & Parenchymal Telangiectasias & Intrahepatic Shunts & Large Confluent Vascular Masses & Biliary Diseases & $\begin{array}{c}\text { Abnormal Origin of Hepatic } \\
\text { Artery }\end{array}$ \\
\hline 1 & + & $A-V$ & - & Biloma & RHA from SMA \\
\hline 2 & + & $A-V$ & - & Slight ectasia & - \\
\hline 3 & + & $A-V$ & - & Biloma & ALHA from LGA \\
\hline 4 & + & $\mathrm{A}-\mathrm{V}$ & - & Slight ectasia & - \\
\hline 5 & + & $A-V$ & - & Slight ectasia & LHA from LGA \\
\hline 6 & + & $\mathrm{A}-\mathrm{V}$ & - & Biloma & - \\
\hline 7 & + & A-P & + & - & RHA from SMA \\
\hline 8 & + & A-P & - & - & - \\
\hline 9 & + & P-V & + & Slight ectasia & - \\
\hline 11 & + & P-V & - & - & - \\
\hline 12 & + & $P-V$ & - & - & - \\
\hline
\end{tabular}

Abbreviations: ALHA, accessory left hepatic artery; A-P, arterioportal shunt; A-V, arteriovenous shunt; LGA, left gastric artery; LHA, left hepatic artery; P-V, portovenous shunt; RHA, right hepatic artery; SMA, superior mesenteric artery.

unit), normal total bilirubin (TBil), and an accompanying intrahepatic arteriovenous shunt.

The remaining 5 patients had slight bile duct ectasia near the porta hepatis without abnormal ALP and TBil levels. Three of them had arterioportal shunts and 2 had portal venous shunts.

\subsubsection{Vascular Anatomic Variants}

Anatomic variants of the hepatic arterial supply were detected in 4 patients. A right hepatic artery originating from the superior mesenteric artery was evident in 2 patients (Figure 5), and a left hepatic artery and an accessory left hepatic artery were observed as originating from the left gastric artery in 2 patients.

\subsubsection{Liver Biopsy Pathology}

Only 1 patient underwent liver biopsy, which was characterized by fibrous tissue and capillary hyperplasia, hepatic cord atrophy, hyperplastic vascular ectasia, and angiomatous disorders (H \& E 200×) (Figure 6). 

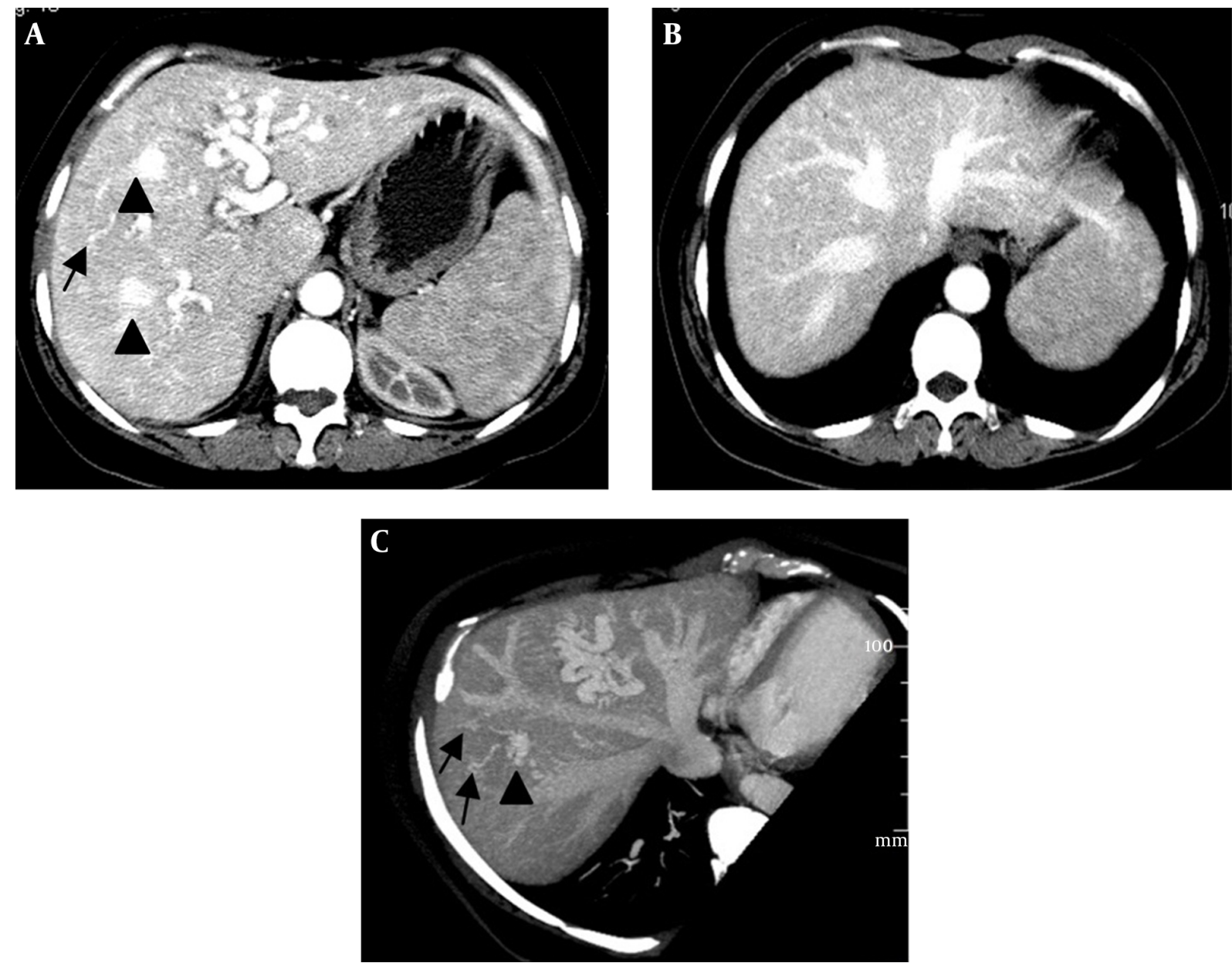

Figure 1. Intrahepatic arteriovenous shunts. A and B, Contrast-enhanced CT shows a dilated hepatic artery and early opacification of the main hepatic veins during the arterial phase; C, Oblique maximal intensity projection MDCT in the parasagittal plane shows a dilated hepatic artery and early simultaneous opacification of the hepatic veins; A and C, Large confluent vascular masses (black arrowheads) and multiple telangiectasias (black arrows) in the peripheral parenchyma are visible.

\section{Discussion}

HHT, or Osler-Weber-Rendu disease, is an autosomal dominant disorder characterized by angiodysplastic lesions (telangiectasias and arteriovenous malformations) that may involve many organs (7). Repeated intractable epistaxis is the main clinical manifestation of HHT. It has been reported that about 93\% patients with HHT sustain epistaxis that is ingravescent with age (8). Sometimes, hemoptysis, melena, hematuresis, fundus hemorrhage, and hypermenorrhea can also be observed. Telangiectasias are observed in about $25 \%$ patients with HHT, with partially revealed multi-organ involvement, such as in the chest, abdomen, and encephalic region.

The incidence of HHT has gradually been increasing in recent years due to the rapid evolution of MDCT technology. MDCT is capable of clearly displaying vascular lesions and hemorrhage within substantive viscera, especially the corresponding post-processing technology, such as MIP. Therefore, it can facilitate in diagnosing the hepatic vascular abnormalities and the various types of shunting (9). The reasons underlying epistaxis had remained unknown for all patients in our study before MDCT, until review of their medical histories was performed in order to determine the diagnosis of HHT.

Liver is considered to be the most commonly involved organ in HHT (4). The prevalence of hepatic involvement in HHT was estimated to be between 8 - 31\% by retrospective studies with single-detector CT (4). However, more recent studies with multidetector CT for consecutive patients have demonstrated that its prevalence is much higher, ranging from about $74-79 \%(3,5)$. Nearly half or even a majority of the patients with HHT were asymptomatic (10). 

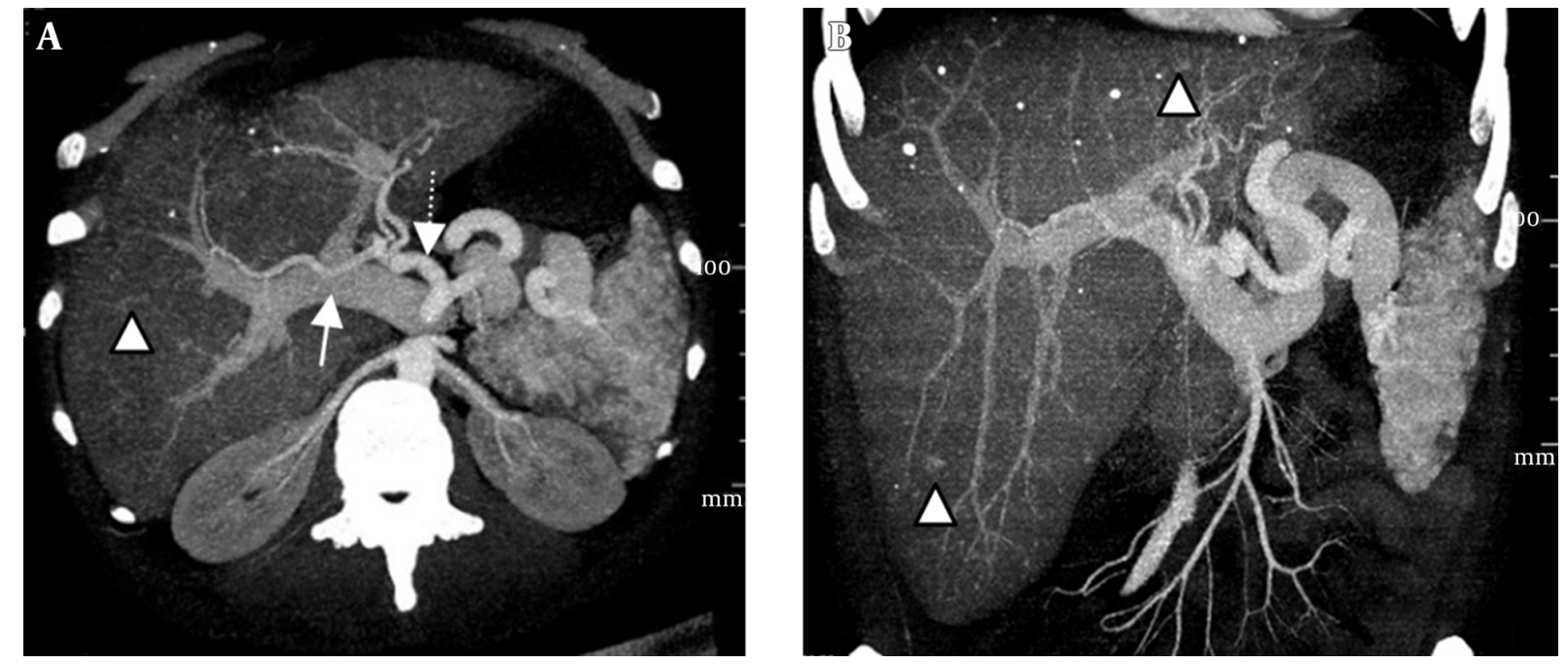

Figure 2. Intrahepatic arterioportal shunts. A and B, Reconstructed images of maximal intensity projection in the parasagittal and coronal plane show early opacification of the portal veins (white arrow) and arteries (dashed arrow). The presence of multiple telangiectasias (white arrowheads) is evident. (Note: These tiny dense highlighted foci in both images, near the described telangiectasias and even outside of the hepatic field, are calcifications.)
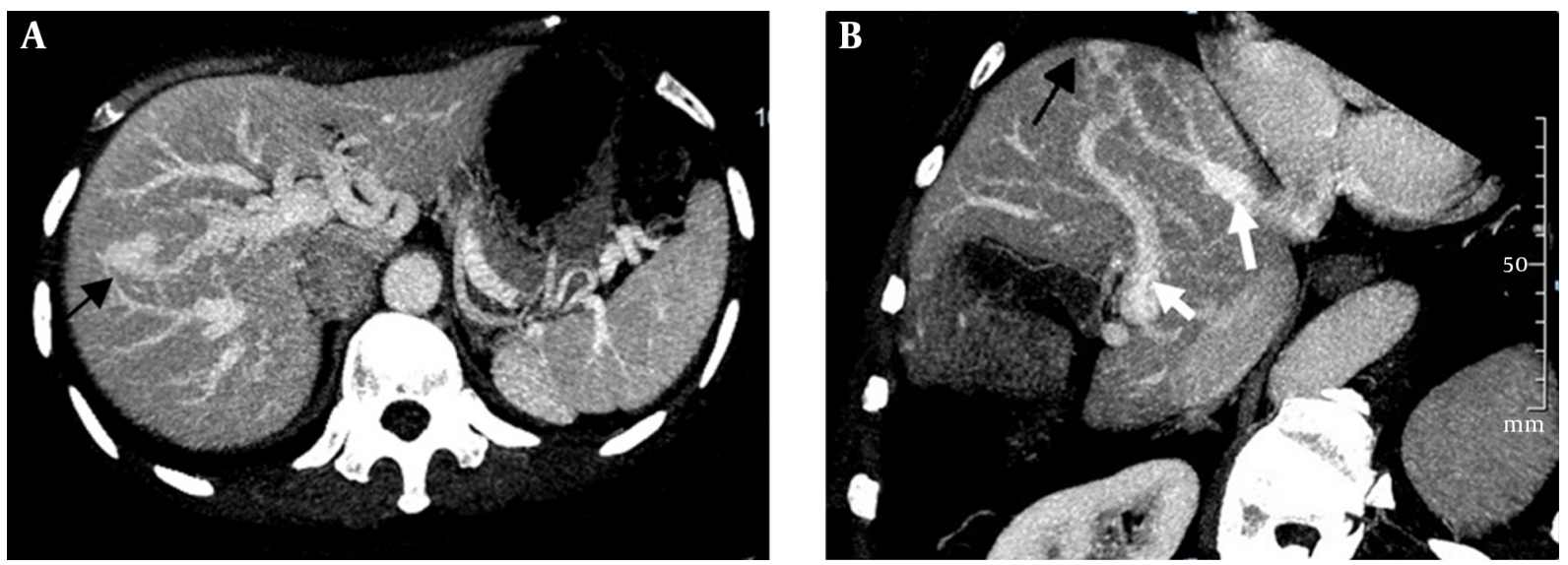

Figure 3. Intrahepatic portal venous shunts. A, Axial; B, Oblique maximal intensity projection images obtained in the venous phase show portal venous shunting, with the dilated portal vein (short white arrow) communicating with the hepatic vein (long white arrow) through a focal vascular mass (black arrow).

Ianora et al. (3) assessed the liver condition of 70 patients with HHT using multiphasic CT and found liver abnormalities in 52 patients in CT scans. However, only 4 of the 52 patients were symptomatic. In our group, most of the patients experienced clinical symptoms of abdominal distension, right upper quadrant abdominal pain, and fatigue, with no specificity. Additionally, the single-involvement of the liver was rare in the HHT patients, and other visceral involvements were concurrent with an incidence rate of approximately $66.7 \%$ in our group.

In our study, one major discovery via liver scanning was the detection of intrahepatic shunting, which can be divided into 3 types: 1) Arteriovenous shunt is the most common. A large quantity of arteriovenous shunts may induce congestive heart failure, leading to hepatomegaly, pulmonary hypertension, and heart failure. Long-term liver ischemia and hypoxia may cause liver fibrosis and hepatic cirrhosis. The incidence of arteriovenous shunts in our group was about 50\%, and these patients typically suffered from fatigue and cyanosis with no hepatic cirrhosis after mild physical activity. CT scanning revealed liver hypertrophy and contrast enhanced CT indicated dilation and tortuosity of the hepatic arteries, as well as early opacification of the main hepatic veins during the arte- 

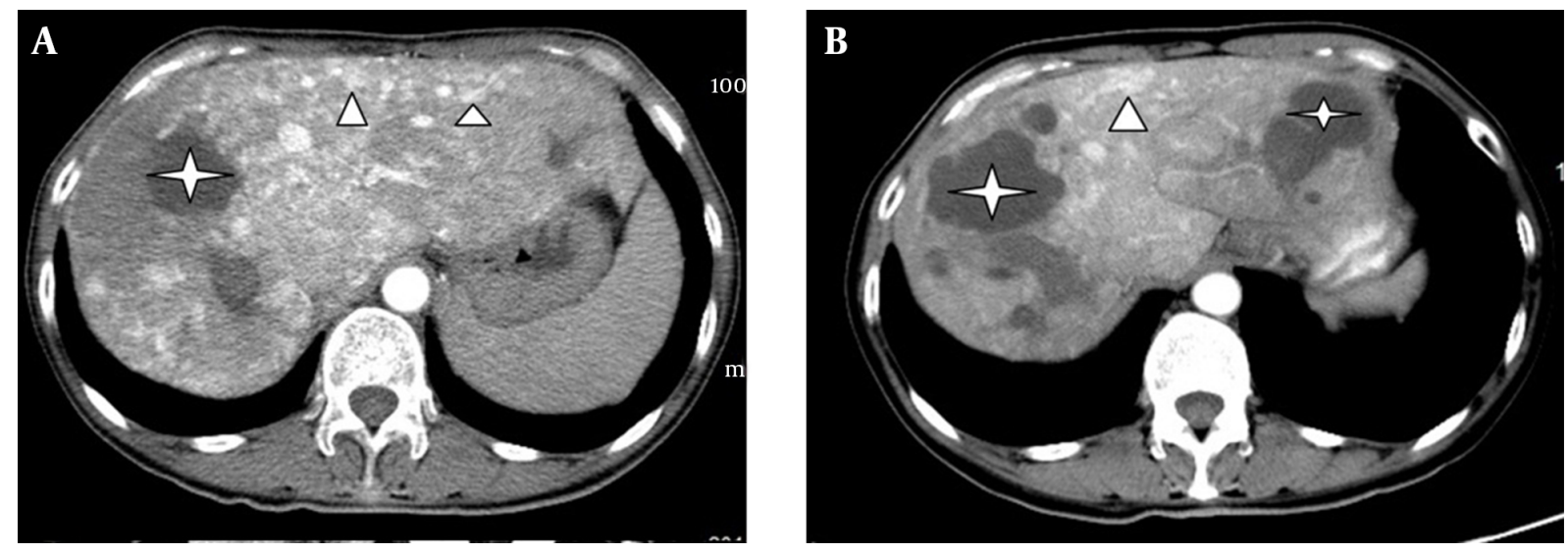

Figure 4. Biliary disease. A, A large biliary cyst lake in the right hepatic lobe (star) and multiple telangiectasias in the peripheral parenchyma (white arrowheads) are detected $\mathrm{B}$, An intrahepatic cystic lesion (star) is shown to be larger after 8 months without any treatment.

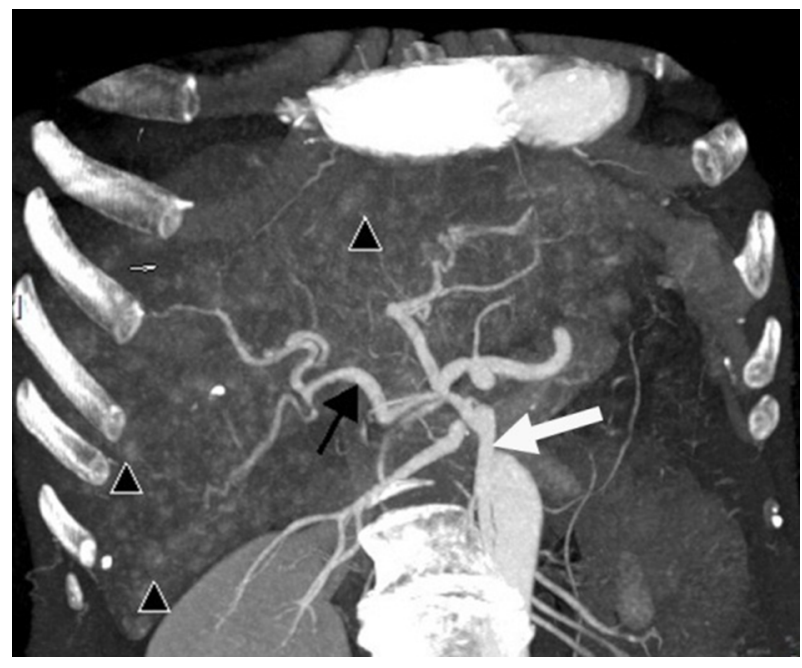

Figure 5. Vascular anatomic variants. The coronal multiplanar reformatted image demonstrates the origin of the right hepatic artery (black arrow) from the superior mesenteric artery (white arrow) and diffuse telangiectasias (black arrowheads) in the peripheral parenchyma.

rial phase; 2) Arterioportal shunts rarely occur according to the literature, and when they do, they are always accompanied by arteriovenous shunts. It has been demonstrated that they are related to portal hypertension and corresponding complications, such as hemorrhage of the digestive tract or abdominal dropsy. Contrast enhanced CT scans revealed early opacification of the portal vein during the arterial phase in 2 cases in our study; 3 ) Portovenous shunt has been rarely reported because it can hardly be detected by routine scanning or angiography, except by microscopy $(10,11)$. However, it has been reported that in an advanced stage of involvement, large portal venous shunts

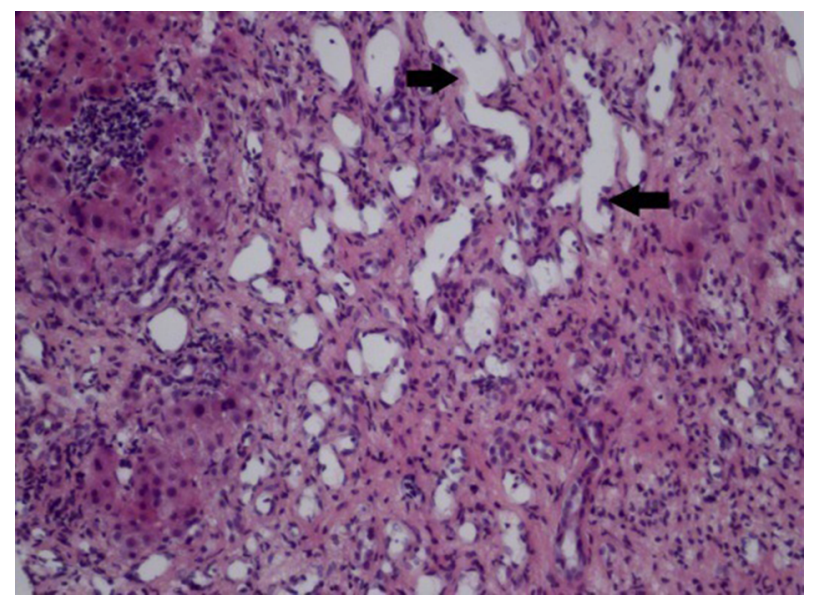

Figure 6. Fibrous tissue and capillary hyperplasia are present, with surrounding hepatic cord atrophy. New vascular structures (black arrow) are shown to be dilated as angiomatous changes $(\mathrm{H} \& \mathrm{E} \times 200)$.

are more likely to be present than the other types of shunts, and the patients with portal venous shunts are older than those without portal venous shunts (12). In our study, dilated portal veins and hepatic veins were revealed during the venous phase in 4 cases, and tortuous ectatic vasculars were clearly observed between the portal vein and the hepatic vein in MIP images. We therefore speculated that MIP might be advantageous in revealing intrahepatic vascular lesions. According to these radiological findings, the possibility of the development of arteriovenous shunts and arterioportal shunts has been ruled out. Due to the elderly age of these patients (all aged 42-68 years), age is less likely to be related to the shunt types. In addition, it was revealed that MDCT and the reconstruction programs such as MIP 
are more favorable in identifying and characterizing the complex vascular alterations typical of HHT (13).

Apart from the shunting, telangiectasias and large confluent vascular masses are also important early findings of the intrahepatic vascular variations, with incidence rates of about $66.7 \%$ and $13.3 \%$, respectively (9). Moreover, abnormal parenchymal perfusion was reported to occur in the later period of HHT with arterioportal shunts. Such a finding was not detected in our study, which was speculated to be related to the course of HHT.

The incidence of the subtype of intrahepatic biliary disease with the liver involved in HHT was high (14), with the main manifestations of biliary stenosis and local cystic dilation $(14,15)$. In our group, 8 out of 12 cases were detected with biliary diseases. The reasons underlying bile duct abnormalities may be the pressure caused by tortuous ectatic vasculars $(16,17)$. For instances, the 5 cases in our group had slight bile duct ectasy near the porta hepatis, and these patients were all asymptomatic with no elevated ALP. However, serious biliary abnormalities occurred due to biliary ischemia, which was induced by intrahepatic shunting. For instances, the 3 cases in our group had circular or tubular bile cysts. In the literature, it has been reported that hepatic artery stenosis after liver transplantation or blockage may also lead to biliary ischemia and necrosis (18), which is in agreement with our findings. Arteriovenous shunts were found in the 3 patients with biliary diseases, indicating that different types of shunting might lead to different degrees of biliary ischemia, and arteriovenous shunting might induce the most serious ischemia, which increases the risk of serious biliary diseases.

In most of the previous reports, these biliary diseases have been described as biliary cysts. However, we believe that the biloma may be a better nomination for the cystic lesions due to the absence of clinical symptoms of cholangiectasis and jaundice. The obvious growth of the biloma after 8 months without treatment in one patient suggested that the biliary diseases progress along with aggravating biliary ischemia. A study reported by Wu et al. proved that biliary diseases occur in the late period of HHT, and its occurrence is closely associated with liver ischemia (17).

Vascular anatomic variants were detected in about $33.3 \%$ of patients ( 4 cases) in our group, which is higher than that reported in previous literature. Three of the 4 cases were detected with accompanying arteriovenous shunts, and the other case with an accompanying portal venous shunt. However, it is unlikely that the relationship between the shunt types and HHT can be precisely defined (17). Still, the data about the anatomic variants of hepatic vasculature is of significance in preoperative assessment, such as in determining candidates for liver transplantation.
Liver biopsy was performed in only one patient in our study, and abnormal ectatic vessels were observed in the specimens of the biopsy. Although it facilitates the diagnosis of intrahepatic vascular disorders, the diagnosis of HHT is best defined based on both clinical and radiological examinations. Given the negative findings by histological examination of the liver in establishing the presence of liver involvement or in classifying the type of liver disease in patients with HHT, this potentially risky procedure is unnecessary (18).

In conclusion, liver involvement by HHT is primarily characterized by diffuse liver vascular shunting and biliary diseases. Specifically, arteriovenous shunts are more likely to induce biloma, and biloma may progress along with aggravating biliary ischemia.

\section{Acknowledgments}

Thanks to all the authors and the above foundations.

\section{Footnotes}

Authors' Contribution: Wenyan Song, clinical data and general writing; Dawei Zhao, imaging analysis; Hongjun Li, clinical imaging and diagnosis; Jinli Ding, imaging and data analysis; Ning He, imaging; Yu Chen, clinical data collection and revision.

Financial Disclosure: None declared.

Funding/Support: This research was supported by the Beijing natural science foundation(No.7132108), the capital health research and development special fund (No. 20112018-01), and the Beijing municipal administration of hospitals clinical medicine development of special funding support (No. ZYLX201511).

\section{References}

1. Dakeishi M, Shioya T, Wada Y, Shindo T, Otaka K, Manabe M, et al. Genetic epidemiology of hereditary hemorrhagic telangiectasia in a local community in the northern part of Japan. Hum Mutat. 2002;19(2):140-8. doi: 10.1002/humu.10026. [PubMed: 11793473].

2. Kjeldsen AD, Vase P, Green A. Hereditary haemorrhagic telangiectasia: a population-based study of prevalence and mortality in Danish patients. J Intern Med. 1999;245(1):31-9. [PubMed: 10095814].

3. Ianora AA, Memeo M, Sabba C, Cirulli A, Rotondo A, Angelelli G. Hereditary hemorrhagic telangiectasia: multi-detector row helical CT assessment of hepatic involvement. Radiology. 2004;230(1):250-9. doi: 10.1148/radiol.2301021745. [PubMed: 14645886].

4. Bernard G, Mion F, Henry L, Plauchu H, Paliard P. Hepatic involvement in hereditary hemorrhagic telangiectasia: clinical, radiological, and hemodynamic studies of 11 cases. Gastroenterology. 1993;105(2):482-7. [PubMed: 8335203].

5. Piskorz MM, Waldbaum C, Volpacchio M, Sorda J. [Liver involvement in hereditary hemorrhagic telangiectasia]. Acta gastroenterologica Latinoamericana. 2011;41(3):225-9. 
6. Shovlin CL, Guttmacher AE, Buscarini E, Faughnan ME, Hyland RH, Westermann CJJ, et al. Diagnostic criteria for hereditary hemorrhagic telangiectasia (Rendu-Osler-Weber syndrome). Am J Med Genetics. 2000;91(1):66-7.

7. Guttmacher AE, Marchuk DA, White RJ. Hereditary hemorrhagic telangiectasia. $N$ Engl J Med. 1995;333(14):918-24. doi: 10.1056/NEJM199510053331407. [PubMed: 7666879].

8. Shovlin CL, Letarte M. Hereditary haemorrhagic telangiectasia and pulmonary arteriovenous malformations: issues in clinical management and review of pathogenic mechanisms. Thorax. 1999;54(8):71429. [PubMed: 10413726]

9. Siddiki H, Doherty MG, Fletcher JG, Stanson AW, Vrtiska TJ, Hough DM, et al. Abdominal findings in hereditary hemorrhagic telangiectasia: pictorial essay on 2D and 3D findings with isotropic multiphase CT. Radiographics. 2008;28(1):171-84. doi: 10.1148/rg.281075037. [PubMed: 18203937].

10. Carette MF, Nedelcu C, Tassart M, Grange JD, Wislez M, Khalil A. Imaging of hereditary hemorrhagic telangiectasia. Cardiovasc Intervent Radiol. 2009;32(4):745-57. doi: 10.1007/s00270-008-9344-2. [PubMed: 18972161].

11. Saluja S, White RI. Hereditary hemorrhagic telangiectasia of the liver: hyperperfusion with relative ischemia-poverty amidst plenty. $R a$ diology. 2004;230(1):25-7. doi: 10.1148/radiol.2293031482. [PubMed: 14695384].

12. Hashimoto M, Tate E, Nishii T, Watarai J, Shioya T, White RI. Angiography of hepatic vascular malformations associated with hereditary hemorrhagic telangiectasia. Cardiovasc Intervent $\mathrm{Ra}$ diol. 2003;26(2):177-80. doi: 10.1007/s00270-002-1507-y. [PubMed:
12813620].

13. Memeo M, Stabile Ianora AA, Scardapane A, Suppressa P, Cirulli A, Sabba C, et al. Hereditary haemorrhagic telangiectasia: study of hepatic vascular alterations with multi-detector row helical CT and reconstruction programs. Radiol Med. 2005;109(1-2):125-38. [PubMed 15729193].

14. Garcia-Tsao G, Korzenik JR, Young L, Henderson KJ, Jain D, Byrd B, et al. Liver disease in patients with hereditary hemorrhagic telangiectasia. NEngl J Med. 2000;343(13):931-6. doi: 10.1056/NEJM200009283431305. [PubMed: 11006369].

15. Rilling WS, Wible BC. SIR 2004 Film Panel Case: hereditary hemorrhagic telangiectasia of the liver with mesenteric steal. J Vasc Interv Radiol. 2004;15(12):1375-9. doi: 10.1097/01.RVI.0000146715.46511.73. [PubMed: 15590791].

16. Kjeldsen $\mathrm{AD}$, Kjeldsen J. Gastrointestinal bleeding in patients with hereditary hemorrhagic telangiectasia. Am J Gastroenterol. 2000;95(2):415-8. doi: 10.1111/j.1572-0241.2000.01792.x. [PubMed: 10685743]

17. Wu JS, Saluja S, Garcia-Tsao G, Chong A, Henderson KJ, White RJ. Liver involvement in hereditary hemorrhagic telangiectasia: CT and clinical findings do not correlate in symptomatic patients. $A J R$ Am J Roentgenol. 2006;187(4):W399-405. doi: 10.2214/AJR.05.1068. [PubMed: 16985112].

18. Zajko AB, Campbell WL, Logsdon GA, Bron KM, Tzakis A, Esquivel $\mathrm{CO}$, et al. Cholangiographic findings in hepatic artery occlusion after liver transplantation. AJR Am J Roentgenol. 1987;149(3):485-9. doi: 10.2214/ajr.149.3.485. [PubMed: 3303874]. 